\title{
Data and Corporate Governance in Pharma and Digital Health: A Necessary Regulatory Convergence
}

\author{
Carter, A.R. ${ }^{1}$, Meinert, E. ${ }^{1}$ \& Brindley, D.A. ${ }^{1 *}$ \\ ${ }^{1}$ University of Oxford, Healthcare Translation Research Group, Department of Paediatrics, Oxford, United \\ Kingdom
}

Authors for Correspondence: david.brindley@paediatrics.ox.ac.uk and alison.carter@paediatrics.ox.ac.uk

Word Count: 1768

\section{Digitalisation of the Pharmaceutical Industry}

The contemporary pharmaceutical industry has its origins in the fine chemicals industry, including dyes and chemical refining. Biotechnology can trace its roots back to brewing, food processing and rudimentary medical practice. Medical devices - including diagnostics - is an evolution of metal working, consumer electronics and, in some cases, providers of primitive surgical tools for barbers. This unlikely constellation of trades, services and technologies is the foundation of the contemporary pharmaceutical industry. This healthcare tapestry is an act of a continuous and ongoing evolution, as are the regulations which govern it.

The regulations that ensure the safety and efficacy of most healthcare products and services - including therapeutics and devices - were stimulated by perceived public health risks in the meatpacking and agricultural industries. Thereafter, this approach with public health at its core was retooled into a technologyspecific approach, focussing on the manufacture of specific substances (later coined as 'drugs'), which at the time was regulated by a nebulous informal collaboration between separate US states and the professional bodies representing their practising clinicians, pharmacists and chemists. In 1906, via the 'Wiley Act,' Theodore Roosevelt enshrined the Food and Drug Act, delegating responsibility for monitoring drug quality to the USDA Bureau of Chemistry. Later, this was formalised into a federal regulatory body, then known as the Food, Drug, and Insecticide Organization - today, known as the FDA. This evolution in the scope of regulatory bodies, driven by technological progress and the public health need, is presently subject to a period of accelerated evolution. This is driven by data.

Change in the pharmaceutical industry is being driven by a trinity of patient need, technological advancement and history, for example, from lessons learnt from the management of existing patient needs in terms of clinical characteristics and/or characteristics of technologies used to treat patient needs. Data is now the most dominant theme impacting each of the three components. Digitisation poses new and unchartered challenges for those responsible with corporate governance in pharma.

Digitisation is pervasive throughout every aspect of our lives - in some cases this is adopted voluntarily, for example in the case of social media, and in others it is instituted on a compulsory basis. The largest and most complex industries, such as healthcare, tend to exhibit the most resistance to change of any type, due to the tremendous 'activation energy' to effect change. These are counterbalanced by the potential significant benefits of technological progress. To use an analogue analogy, in the past decade, members of the healthcare sector have been listening to one 'data vinyl' - with both an A and B Side.

In this analogy, the A Side would be conventional pharma and devices, and business as usual through novel therapeutic and diagnostic technologies, with limited voluntary participation in digital innovation(s). Meanwhile, our B Side represents the digital health sector has sought independence from conventional pharma, not least from its perceived conservative attitudes and complex regulatory environment.

Fortunately for patients - but to the alarm of those responsible for the corporate governance of pharmaceutical and digital health organisations - both audiences are now being forced to adopt the harmony of the regulated digital pharmaceutical industry. 
Pharma, akin to elderly relatives being forced to sign up to social media so they can 'check in' at locations to access complementary WIFI, is being forced to engage with the 'big data' agenda. Similarly, digital health startups - often staffed with individuals from non-regulated and non-healthcare backgrounds, are being forced to engage in GxP regulations enforced by National Competent Authorities (NCAs) such as the FDA. This can be a uncomfortable pill to swallow for people used to working in sectors with the freedoms of minimal regulation.

The unexpected convergence of these diametrically opposed working practices and cultures (i.e. digital health and pharmaceutical companies) has created some minor confusion and consternation amongst senior management responsible for the operations and strategy of these organisations. However, boardrooms around the world are characterised by a sense of confusion and uncertainty at best, or abject alarm and existential crisis at worst, in regard to how to implement digitisation in accordance with necessary GXP regulated standards effectively. Gone are the delusion and the days when it was thought that blissful ignorance was an acceptable response, or that a state of denial regarding the digitisation of the pharmaceutical industry could continue. All are aware that the costs of getting GxP regulation of data in pharma are immense and for some companies will be fatal.

In the past, data security and integrity were board policies reviewed briefly and annually, often deep within an Audit Committee agenda. In the wake of Cambridge Analytica, EU General Data Protection Regulation (GDPR), US HIPPA, phishing attacks, ransomware, malware, a crippling NHS cyber-attack, FDA Digital Health Software Precertification (Pre-Cert) Program, increased NCA scrutiny data integrity compliance (to 21 CFR Part 11 and Eudralex Chapter 4, Annex 11), this cursory periodic review of data standards is insufficient.. However, BoD members - of pharma, device and digital health companies - should not assume 'no news is good news' or only respond reactively to data crises. Proactive corporate governance to ensure GxP alignment of all organisational processes, services, equipment and vendors is essential to reduce the risk of a data crisis and provide a robust foundation to mount corrective actions if needed. These challenge raise the question of scope.

\section{Horizontal Integration of Therapeutics, Devices and Digital Health}

Historically pharmaceutical companies were standalone in their structure. That is to say, self-sufficient, providing the majority of products and services required to meet end-user needs via internal resources. Therein, the majority of compliance issues could be categorised into the following: (1) internal [specific to company sites]; (2) external (product or service) [relating to the implementation of a company's products or services by the end user, off site]; or (3) external (support) [outsourced functions]. The last would have been limited in utilisation and therein risk.

In common with other industries and further enforced by the now established trend of outsourced pharma R\&D and growth through acquisition, pharma moved to adopt a virtually integrated business model. For example, previous proprietary manufacturing capabilities have been outsourced to Contract Manufacturing Organisations (CMOs). This in turn shifted the balance of internal versus external operations - including relating to data management - driving a significant increase in 'critical supplier audit burden' for quality and regulatory functions. Therefore, vertical integration in the pharmaceutical industry increased the complexity and risks of GxP data management. However, today, horizontal integration is further expanding the scope of the data management risks driven by regulatory changes and increasing blurred lines of product classifications.

Previously, clear product-based distinctions could be drawn between organisations between therapeutics [required to demonstrate efficacy and safety], devices [required to demonstrate safety and equivalence] and digital health technologies which did not have any formal regulatory requirements. Therein, acceptance standards for therapeutics and devices were determined and assessed by independent bodies, National Competent Authorities (NCAs) and Notified Bodies (NBs). Conversely, acceptance standards for digital health technologies were predominantly adopted from non-healthcare sectors, with acceptance criteria determined by end users. A horizontal consolidation of technology classifications and therein acceptance criteria is occurring today.

The regulatory landscape for medical devices has evolved to be in closer alignment with therapeutics, driven by a necessity for greater evidence of device effectiveness and criticality of (companion) diagnostics to clinical care. 
More recently, digital health has been subject to increased regulatory visibility by NCAs, aligned increasingly closely with medical device regulations. This is due to increasing complexity and risk of digital health technologies - particularly those which inform or replace clinical decision making. Therefore, there is increasing uncertainty in regard to the standards and regulations to which boards should be implementing governance in healthcare.

Central to cGMP manufacturing processes for therapeutics and devices is the principle of Quality by Design $(\mathrm{QbD})$. That is to say that all aspects of a manufacturing processes should be designed to maintain and minimise risk to critical quality attributes (CQAs) of end products. These authors propose that corporate governance of pharmaceutical, medical device and digital health companies should be subject to a de novo, documented and continuous process of Governance by Design $(\mathrm{GbD})$, with data management to appropriate standards at its heart.

Responsibility and accountability for data management in healthcare can no longer be siloed by function within an organisation (for example, to the Data Protection Officer), nor by product or service. All functions and all products and services within healthcare are now subject to two types of data governance requirement: GxP and non-GxP. These authors assert that very few board members of healthcare organisations accountable for corporate governance, and data governance therein, have a sufficient understanding of this distinction of its implications. This is part due to the rapid horizontal integration of data gathering and data deployment in the sector.

Non-GxP data governance requirements include standard legal requirements to which all companies, not solely healthcare companies, are subject to; for example, relating to the protection and management of personal data. These are monitored and enforced by public bodies - with responsibility for all sectors - which have delegated authority from governments. Conversely, GxP data governance requirements apply solely to organisations operating in the healthcare sector - of which the digital health industry is now part - and is subject to the purview of National Competent Authorities, including the FDA.

Therefore, the concurrent horizontal integration of pharmaceutical, medical device and digital health regulatory standards into an increasingly coherent, self-contained and harmonised corpus of NCA-enforced GxP regulations has yielded a new operational reality for healthcare organisations. Alongside this, the deeper penetration of non-GxP data governance standards into vertically integrated functions has created new requirements for healthcare corporate governance.

Independent of your organisation's offering - therapeutic, device or software - those responsible for corporate governance must assume that every data driven aspect of operations is subject to GxP regulations and the (pragmatic) purview of the FDA. Data flows in healthcare are horizontal and vertical. Consequently, and rightly, so now is GxP regulation.

Pharma is in the business of data; and data is in the business of pharma. Corporate governance in your organisation must reflect this. 